\title{
Comparative evaluation of toric intraocular lens alignment and visual quality with image- guided surgery and conventional three-step manual marking
}

This article was published in the following Dove Press journal: Clinical Ophthalmology

\author{
Jeewan S Titiyal \\ Manpreet Kaur \\ Cijin P Jose \\ Ruchita Falera \\ Ashutosh Kinkar \\ Lalit MS Bageshwar
}

Cornea, Cataract and Refractive Surgery Services, Dr Rajendra Prasad Centre for Ophthalmic Sciences, All India Institute of Medical Sciences, New Delhi, India
Correspondence: Jeewan S Titiyal

Cornea, Cataract and Refractive Surgery Services, Dr Rajendra Prasad Centre for Ophthalmic Sciences, All India Institute of Medical Sciences, Ansari Nagar, New Delhi - II 0029, India Tel +9| || 26593 |46 Email titiyal@gmail.com
Purpose: To compare toric intraocular lens (IOL) alignment assisted by image-guided surgery or manual marking methods and its impact on visual quality.

Patients and methods: This prospective comparative study enrolled 80 eyes with cataract and astigmatism $\geq 1.5 \mathrm{D}$ to undergo phacoemulsification with toric IOL alignment by manual marking method using bubble marker (group $\mathrm{I}, \mathrm{n}=40$ ) or Callisto eye and $\mathrm{Z}$ align (group II, $\mathrm{n}=40$ ). Postoperatively, accuracy of alignment and visual quality was assessed with a ray tracing aberrometer. Primary outcome measure was deviation from the target axis of implantation. Secondary outcome measures were visual quality and acuity. Follow-up was performed on postoperative days (PODs) 1 and 30.

Results: Deviation from the target axis of implantation was significantly less in group II on PODs 1 and 30 (group I: $5.5^{\circ} \pm 3.3^{\circ}$, group II: $3.6^{\circ} \pm 2.6^{\circ} ; p=0.005$ ). Postoperative refractive cylinder was $-0.89 \pm 0.35 \mathrm{D}$ in group I and $-0.64 \pm 0.36 \mathrm{D}$ in group II $(p=0.003)$. Visual acuity was comparable between both the groups. Visual quality measured in terms of Strehl ratio $(p<0.05)$ and modulation transfer function (MTF) $(p<0.05)$ was significantly better in the image-guided surgery group. Significant negative correlation was observed between deviation from target axis and visual quality parameters (Strehl ratio and MTF) $(p<0.05)$.

Conclusion: Image-guided surgery allows precise alignment of toric IOL without need for reference marking. It is associated with superior visual quality which correlates with the precision of IOL alignment.

Keywords: toric IOL alignment, image-guided surgery, manual marking toric IOL, Callisto eye and $\mathrm{Z}$ align toric IOL, visual quality toric IOL, visual quality image-guided surgery

\section{Introduction}

Corneal astigmatism of $1 \mathrm{D}$ or more may be present in more than one-third of the patients undergoing cataract surgery, and toric intraocular lenses (IOLs) are a safe and effective modality in such cases to help achieve emmetropia. ${ }^{1-3}$ Accurate toric IOL alignment along the desired corneal meridian is a prerequisite for achieving an optimal visual outcome. One degree of misalignment reduces the astigmatic correction by $\sim 3.3 \%$, with more than $30^{\circ}$ misalignment effectively increasing the amount of preoperative astigmatism. ${ }^{4,5}$

A three-step procedure has conventionally been used for axis marking on the cornea and helps in alignment of the IOL within $5^{\circ}$ of the intended axis. ${ }^{6}$ However, the subjective nature of this procedure can lead to inaccurate marking in inexperienced 
hands and result in a suboptimal visual outcome. Several image-guided modalities have been developed for precise toric IOL alignment that obviate the need for preoperative axis marking and aim to decrease the subjectivity associated with manual marking. ${ }^{7-10}$ A superior alignment of the toric IOL has been demonstrated with the image-guided systems as compared to conventional manual marking methods; however, the visual outcomes are comparable. ${ }^{7-9}$ No study has evaluated the effect of toric IOL alignment on visual quality with different marking methods.

We herein compared the toric IOL alignment assisted by Callisto eye and $\mathrm{Z}$ align (Carl Zeiss Meditec AG, Jena, Germany) and manual axis marking using the bubble marker (Nuijts Lane Toric Reference Marker), and its impact on visual quality.

\section{Patients and methods}

The prospective comparative study was conducted at Dr Rajendra Prasad Centre for Ophthalmic Sciences, AIIMS, New Delhi, India. Ethical clearance was obtained from the institutional review board (AIIMS, New Delhi). Written informed consent was obtained from all patients. The study conforms to the tenets of the Declaration of Helsinki.

Eighty patients ( 80 eyes) with cataract and astigmatism $\geq 1.5$ D scheduled to undergo phacoemulsification with toric IOL implantation were enrolled in the study. Eyes with pupil dilation of at least $5.0 \mathrm{~mm}$, axial length $22-24.5 \mathrm{~mm}$, regular astigmatism ranging from 1.5 to $6 \mathrm{D}$, and no significant ocular or systemic co-morbidity were included. Eyes with pre-existing glaucoma, retinal pathology, small pupils ( $<5 \mathrm{~mm}$ ), corneal opacity, ocular surface disorders, history of contact lens usage, and previous ocular surgeries were excluded. Eyes with intraoperative complications such as extension of capsulorhexis, posterior capsular rent, vitreous loss, and nucleus drop were excluded. Eyes that required suture to seal the corneal incision at end of surgery were also excluded.

Patients were enrolled into two groups based on the method of toric IOL alignment. Group I ( $n=40)$ underwent manual marking of the reference and target axes, and group II $(n=40)$ underwent toric IOL alignment using Callisto eye and $\mathrm{Z}$ align. All surgeries were performed by a single surgeon.

Preoperative comprehensive examination was done, including uncorrected distance visual acuity (UDVA), corrected distance visual acuity (CDVA), slitlamp biomicroscopy, fundus examination, applanation tonometery, specular microscopy, manual keratometery (Bausch and Lomb keratometer), videokeratography, IOL master image capture, and optical biometry (IOL Master 500). The emmetropic IOL power was calculated for AcrySof Toric IOL (Alcon Laboratories, Inc., Fort Worth, TX, USA) based on the Alcon online toric IOL calculator (Alcon Laboratories, Inc.). The first-generation Alcon online toric IOL calculator was used for calculations, which did not incorporate the Barrett toric algorithm.

\section{Technique for IOL alignment}

In group I, the reference axis was marked preoperatively at 3, 6, and 9'o clock positions using the bubble marker (Nuijts Lane Toric Reference Marker) with the patient positioned erect and maintaining a straight-ahead gaze. The preoperative marks were placed by a single experienced surgeon in all cases. Intraoperatively, the reference limbal marks were aligned to the degree gauge on the fixation ring (Mendez gauge), and the target axis was marked with a two-ray axis marker inked with marking ink. A $2.2 \mathrm{~mm}$ clear corneal incision was made temporally, and two $1.1 \mathrm{~mm}$ side port incisions were made at $90^{\circ}$ and $240^{\circ}$. Anterior continuous curvilinear capsulorhexis was performed with a $26-\mathrm{G}$ needle cystotome and the intended capsulorhexis diameter was $\sim 5 \mathrm{~mm}$. Co-axial phacoemulsification (Centurion Vision System; Alcon Laboratories, Inc.) was performed and toric IOL (AcrySof SN60TT; Alcon Laboratories, Inc.) was implanted in the bag. The IOL was rotated to align with the target axis that was marked earlier using the axis marker. The IOL was kept $3^{\circ}-5^{\circ}$ anticlockwise after completion of irrigationaspiration and complete removal of ophthalmic viscosurgical device. Stromal hydration of the corneal incisions was performed and the toric IOL alignment was completed.

In group II, preoperative digital reference imaging was obtained by the IOLMaster 500 biometer (Carl Zeiss Meditec AG) to determine the reference axis. This was exported into the Callisto eye system. Intraoperative registration was done with real-time intraoperative eye tracking of limbal and scleral vessels. A graphical overlay served as a visible guide to the surgeon for toric IOL alignment along the desired axis. Co-axial phacoemulsification (Centurion Vision System; Alcon Laboratories, Inc.) was performed and toric IOL was implanted in the bag as in group I. The IOL was aligned to the target axis that was directly projected onto the surgical field.

\section{Postoperative rotational check}

Postoperatively, ray tracing aberrometer (iTrace system; Tracey Technologies, Houston, TX, USA) with the toric IOL enhancement software was used to calculate the resultant 


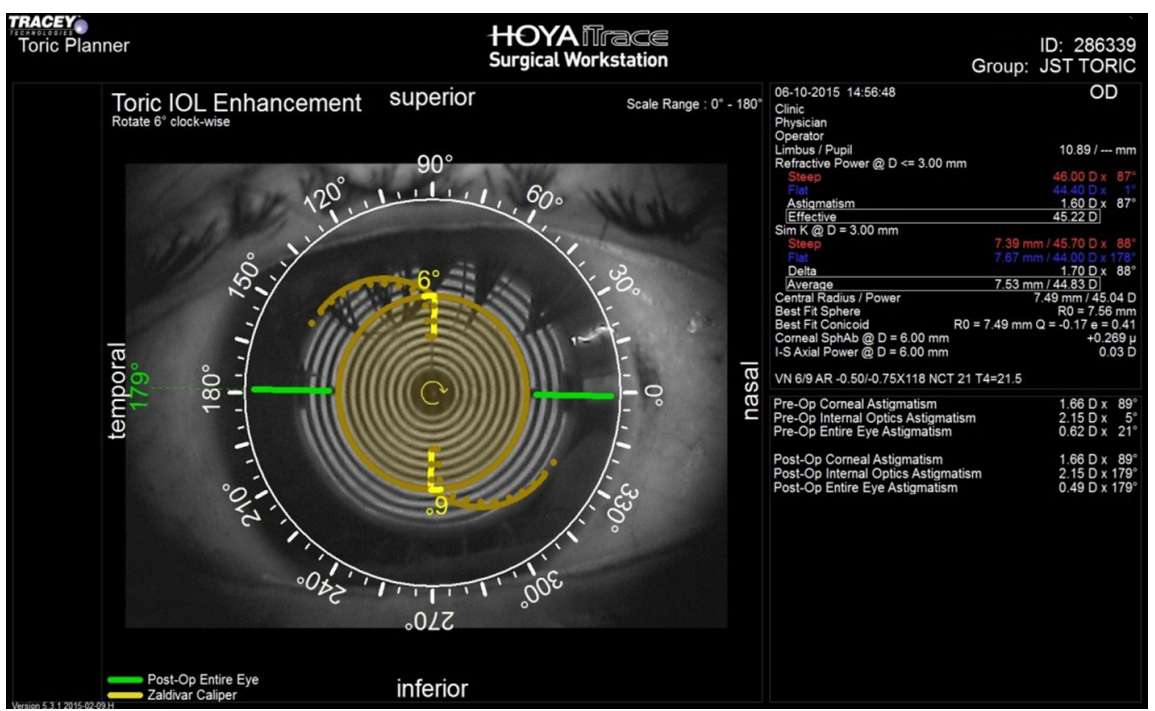

Figure I Postoperative assessment of toric IOL alignment with ray tracing aberrometer in a case in group I (conventional manual marking). A $6^{\circ}$ deviation from target axis of implantation is present.

Abbreviation: IOL, intraocular lens.

toric IOL axis in both groups on postoperative days (PODs) 1 and 30. Angle difference between the target and final axis was also calculated (Figures 1 and 2). The postoperative defocus equivalent, corneal cylinder, refractive sphere, and refractive cylinder were measured with the iTrace system with pupil dilation of at least $5 \mathrm{~mm}$.

The objective assessment of visual quality was performed on the iTrace system, and the modulation transfer function (MTF) and point spread function (PSF) were evaluated. The MTF represents the visual performance of the eye across the range of spatial frequencies used in daily life. The Strehl ratio is the ratio of the peak height of the PSF being measured to the peak height of a perfect optical system and ranges from 0 to 1 , with 1 representing a perfect optical system. ${ }^{11}$ Both internal (lens) and total Strehl ratio and MTF were recorded.

Postoperative UDVA, CDVA, and IOP were recorded in all cases. The primary outcome measure was deviation from target axis of implantation. The secondary outcome measures were visual quality and acuity. The technician performing the iTrace examination was blinded to the method of toric IOL alignment. Follow-up examinations were performed on PODs 1 and 30 .

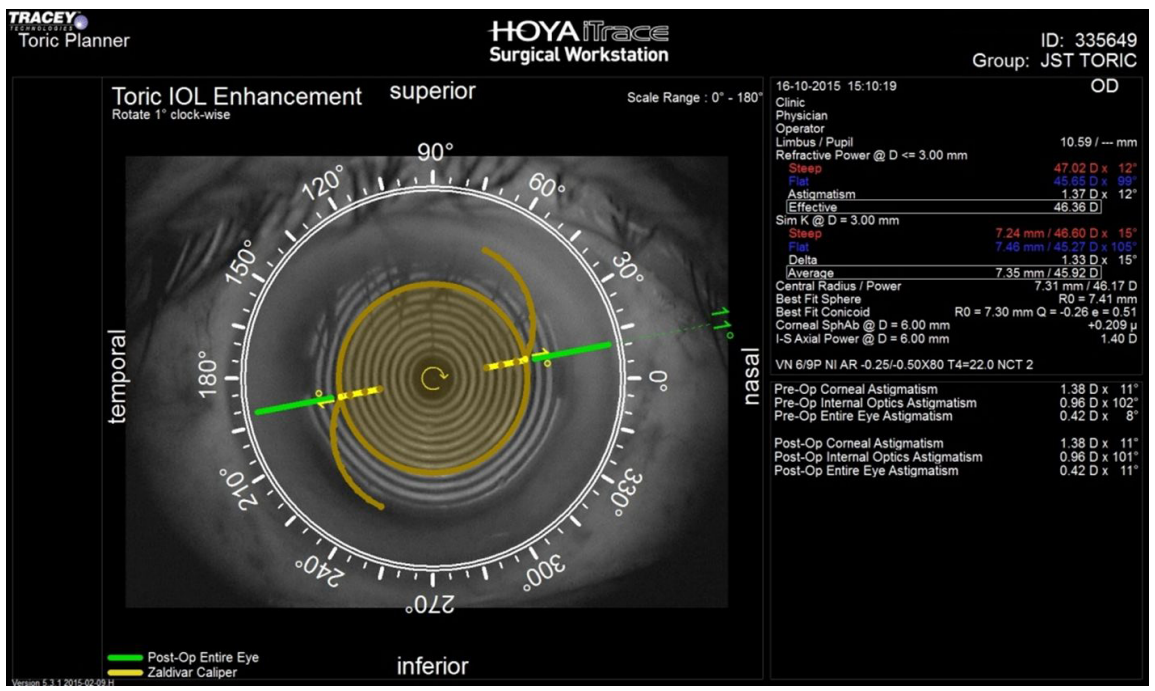

Figure 2 Postoperative assessment of toric IOL alignment with ray tracing aberrometer in a case in group II (image-guided surgery). A I ${ }^{\circ}$ deviation from target axis of implantation is present.

Abbreviation: IOL, intraocular lens. 


\section{Statistical analysis}

Statistical analysis was done using Statistical Package for the Social Sciences (SPSS 11.0; SPSS Inc., Chicago, IL, USA). Normally distributed continuous variables were expressed as mean \pm standard deviation and were compared using independent samples $t$-test. Non-normally distributed continuous variables were compared using Mann-Whitney $U$-test. Nominal data were compared using chi-square test or Fisher's exact test as appropriate. Correlation between the deviation from target axis of implantation and visual quality parameters was assessed using the Pearson's correlation test. $p$-value less than 0.05 was considered significant.

\section{Results}

The demographic details and preoperative characteristics of the cases have been summarized in Table 1 . The mean age of the patients was $63.1 \pm 13.7$ years in group I and $60.1 \pm 10.3$ years in group II $(p=0.271)$. The preoperative corneal cylinder was $2.1 \pm 0.9 \mathrm{D}$ in group I and $2.5 \pm 0.9 \mathrm{D}$ in group II $(p=0.057)$.

Postoperative anatomical and refractive outcomes are summarized in Table 2. Postoperatively, the deviation from target axis of implantation as assessed by the ray tracing aberrometer on POD 1 was $5.8^{\circ} \pm 3.7^{\circ}$ in group I and $3.7^{\circ} \pm 2.8^{\circ}$ in group II ( $p=0.005)$. On POD 30, the deviation from target axis of implantation was $5.5^{\circ} \pm 3.3^{\circ}$ in group I and $3.6^{\circ} \pm 2.6^{\circ}$ in group II $(p=0.005)$.

The postoperative refractive cylinder was $-0.89 \pm 0.35 \mathrm{D}$ in group I and $-0.64 \pm 0.36 \mathrm{D}$ in group II $(p=0.003)$.

Table I Demographic details and preoperative characteristics of cases undergoing toric IOL implantation

\begin{tabular}{llll}
\hline Demographic variables & $\begin{array}{l}\text { Group I } \\
\text { (manual } \\
\text { marking) } \\
\mathbf{N}=\mathbf{4 0}\end{array}$ & $\begin{array}{l}\text { Group II } \\
\text { (Callisto } \\
\text { assisted) } \\
\mathbf{N}=\mathbf{4 0}\end{array}$ & p-value \\
\hline Age (years) & $63.1 \pm 13.7$ & $60.1 \pm 10.3$ & $0.27 \mid$ \\
Sex (M:F) & $17: 23$ & $19: 21$ & 0.822 \\
Right eye: left eye & $24: 16$ & $18: 22$ & 0.263 \\
Preoperative characteristics & & \\
Corneal cylinder (D) & $2.1 \pm 0.9$ & $2.5 \pm 0.9$ & 0.057 \\
Defocus equivalent & $1.2 \pm 2.8$ & $2.0 \pm 3.2$ & 0.211 \\
Keratometry I & $42.6 \pm 1.8$ & $42 \pm 1.8$ & 0.148 \\
Keratometry 2 & $44.6 \pm 1.9$ & $44.4 \pm 2.2$ & 0.724 \\
UDVA (logMAR units) & $0.78 \pm 0.37$ & $0.97 \pm 0.28$ & 0.013 \\
CDVA (logMAR units) & $0.56 \pm 0.38$ & $0.59 \pm 0.29$ & 0.746 \\
IOP (mmHg) & $\mid 4.9 \pm 3.01$ & $14.4 \pm 3.04$ & 0.508 \\
Target axis of implantation & $100.2 \pm 74.8$ & $104.2 \pm 68.8$ & 0.804 \\
\hline Ab &
\end{tabular}

Abbreviations: $M$, male; F, female; D, Diopter; UDVA, uncorrected distance visual acuity; CDVA, corrected distance visual acuity; logMAR, logarithm of minimum angle of resolution; IOP, intraocular pressure.
Table 2 Postoperative visual outcomes and toric IOL alignment in cases undergoing toric IOL implantation with manual marking or image-guided surgery

\begin{tabular}{llll}
\hline Outcome measures & $\begin{array}{l}\text { Group I } \\
\text { (manual } \\
\text { marking) } \\
\mathbf{N}=\mathbf{4 0}\end{array}$ & $\begin{array}{l}\text { Group II } \\
\text { (Callisto } \\
\text { assisted) } \\
\mathbf{N}=\mathbf{4 0}\end{array}$ & p-value \\
\hline Refractive sphere & $0.39 \pm 0.44$ & $0.15 \pm 0.79$ & 0.105 \\
Refractive cylinder & $-0.89 \pm 0.35$ & $-0.64 \pm 0.36$ & $\mathbf{0 . 0 0 3}$ \\
Corneal cylinder & $2.1 \pm 0.9$ & $2.3 \pm 1.2$ & 0.474 \\
Defocus equivalent & $0.01 \pm 0.2$ & $0.07 \pm 0.3$ & 0.208 \\
UDVA (logMAR units) POD I & $0.055 \pm 0.09$ & $0.03 \pm 0.07$ & 0.184 \\
CDVA (logMAR units) POD I & $0.01 \pm 0.03$ & $0.005 \pm 0.02$ & 0.402 \\
UDVA (logMAR units) POD 30 & $0.025 \pm 0.06$ & $0.017 \pm 0.05$ & 0.541 \\
CDVA (logMAR units) POD 30 & $0.008 \pm 0.03$ & $0.005 \pm 0.02$ & 0.649 \\
IOP (mmHg) & $15.2 \pm 2.9$ & $14.4 \pm 2.9$ & 0.272 \\
Axis of IOL implantation & $82.6 \pm 72.3$ & $104.6 \pm 69.0$ & 0.169 \\
Deviation from target axis & $5.8 \pm 3.7$ & $3.7 \pm 2.8$ & $\mathbf{0 . 0 0 5}$ \\
(POD I) & & & \\
Deviation from target axis & $5.5 \pm 3.3$ & $3.6 \pm 2.6$ & $\mathbf{0 . 0 0 5}$ \\
(POD 30) & & & \\
\hline
\end{tabular}

Note: $p$-values less than 0.05 are shown in bold.

Abbreviations: D, Diopter; UDVA, uncorrected distance visual acuity; CDVA, corrected distance visual acuity; logMAR, logarithm of minimum angle of resolution; IOP, intraocular pressure; IOL, intraocular lens; POD, postoperative day.

The postoperative corneal cylinder was $2.1 \pm 0.9 \mathrm{D}$ in group I and $2.3 \pm 1.2 \mathrm{D}$ in group II $(p=0.474)$.

The postoperative UDVA at 1 day was $0.055 \pm 0.09$ logarithm of minimum angle of resolution (logMAR) units in group I and $0.030 \pm 0.07 \log$ MAR units in group II ( $p=0.184)$. The postoperative UDVA at 1 month was $0.025 \pm 0.06$ $\log$ MAR units in group I and $0.017 \pm 0.05 \log$ MAR units in group II $(p=0.541)$.

The visual quality parameters, including the Strehl ratio and MTR, are summarized in Table 3. The internal Strehl ratio was significantly better in the image-guided surgery group on POD 1 (group I: $0.051 \pm 0.056$, group II: $0.109 \pm 0.142 ; p=0.019$ ) and POD 30 (group I: $0.075 \pm 0.081$, group II: $0.149 \pm 0.162 ; p=0.012$ ). The internal MTF was significantly better in the image-guided surgery group at the spatial frequencies of 5, 10, and 15 cycles per degree (cpd), as well as the average MTF (average MTF group I: $0.218 \pm 0.085$, group II: $0.273 \pm 0.115 ; p=0.016$ ). The difference between the two groups persisted at POD 30 as well (average MTF group I: $0.226 \pm 0.102$, group II: $0.282 \pm 0.130 ; p=0.036$ ).

The correlation between the rotational deviation from target axis of IOL implantation and the visual quality parameters is summarized in Table 4. A significant negative correlation was observed between the deviation from target axis of toric IOL alignment on PODs 1 and 30, and the Strehl ratio internal $(p<0.001)$, Strehl ratio total $(p<0.001)$, MTF internal $(p<0.001)$, and MTF total $(p<0.05)$. 
Table 3 Assessment of visual quality in cases undergoing toric intraocular lens alignment with manual marking or image-guided surgery

\begin{tabular}{|c|c|c|c|}
\hline $\begin{array}{l}\text { Visual quality } \\
\text { parameters }\end{array}$ & $\begin{array}{l}\text { Group I } \\
\text { (manual } \\
\text { marking) } \\
\mathbf{N}=\mathbf{4 0}\end{array}$ & $\begin{array}{l}\text { Group II } \\
\text { (Callisto } \\
\text { assisted) } \\
\mathbf{N}=\mathbf{4 0}\end{array}$ & $p$-value \\
\hline \multicolumn{4}{|l|}{ Postoperative day I } \\
\hline Strehl ratio internal & $0.05 I \pm 0.056$ & $0.109 \pm 0.142$ & 0.019 \\
\hline Strehl ratio total & $0.090 \pm 0.104$ & $0.136 \pm 0.182$ & 0.170 \\
\hline MTF internal 5 cpd & $0.344 \pm 0.214$ & $0.484 \pm 0.235$ & 0.007 \\
\hline MTF internal $10 \mathrm{cpd}$ & $0.172 \pm 0.115$ & $0.249 \pm 0.174$ & 0.021 \\
\hline MTF internal I5 cpd & $0.107 \pm 0.072$ & $0.153 \pm 0.121$ & 0.042 \\
\hline MTF internal average & $0.218 \pm 0.085$ & $0.273 \pm 0.115$ & 0.016 \\
\hline MTF total 5 cpd & $0.517 \pm 0.202$ & $0.550 \pm 0.209$ & 0.480 \\
\hline MTF total $10 \mathrm{cpd}$ & $0.249 \pm 0.146$ & $0.273 \pm 0.178$ & 0.508 \\
\hline MTF total I5 cpd & $0.162 \pm 0.103$ & $0.187 \pm 0.119$ & 0.327 \\
\hline MTF total average & $0.282 \pm 0.101$ & $0.302 \pm 0.109$ & 0.406 \\
\hline \multicolumn{4}{|l|}{ Postoperative day 30} \\
\hline Strehl ratio internal & $0.075 \pm 0.081$ & $0.149 \pm 0.162$ & 0.012 \\
\hline Strehl ratio total & $0.101 \pm 0.085$ & $0.134 \pm 0.120$ & 0.160 \\
\hline MTF internal 5 cpd & $0.373 \pm 0.250$ & $0.492 \pm 0.271$ & 0.045 \\
\hline MTF internal I0 cpd & $0.190 \pm 0.142$ & $0.264 \pm 0.186$ & $0.05 I$ \\
\hline MTF internal I5 cpd & $0.110 \pm 0.078$ & $0.165 \pm 0.129$ & 0.023 \\
\hline MTF internal average & $0.226 \pm 0.102$ & $0.282 \pm 0.130$ & 0.036 \\
\hline MTF total $5 \mathrm{cpd}$ & $0.546 \pm 0.179$ & $0.599 \pm 0.215$ & 0.245 \\
\hline MTF total $10 \mathrm{cpd}$ & $0.277 \pm 0.136$ & $0.312 \pm 0.169$ & 0.301 \\
\hline MTF total I5 cpd & $0.197 \pm 0.158$ & $0.212 \pm 0.168$ & 0.675 \\
\hline MTF total average & $0.299 \pm 0.088$ & $0.319 \pm 0.107$ & 0.343 \\
\hline
\end{tabular}

Note: $p$-values less than 0.05 are shown in bold.

Abbreviations: MTF, modulation transfer function; cpd, cycles per degree.

All cases had an adequate IOL coverage of $0.3-0.5 \mathrm{~mm}$ all around by the anterior capsulorhexis margin. The corneal incisions were sealed with stromal hydration at the end of surgery, and a suture-assisted wound closure was not needed in any case. The postoperative course was uneventful, and no case developed severe corneal edema, elevated IOP, or anterior segment inflammation on follow-up. Postoperative toric IOL realignment was not needed in any case.

\section{Discussion}

Patient expectations of the post-surgical refractive outcomes have been increasing ever since the first toric lens implantation in $1994 .{ }^{4}$ New methods are constantly being developed to enhance the precision of toric IOL alignment. We have progressed from the initial free-hand limbal marking to the development of intraoperative abberrometry that will allow real-time adjustment of toric IOL orientation for enhanced visual outcome. ${ }^{7-15}$ Conventional manual marking is still a widely prevalent method of toric IOL alignment, and we compared image-guided surgery with the three-step axis marking using a bubble marker.

We implanted a single-piece toric acrylic IOL (AcrySof SN60TT) in all cases, which is rotationally stable in the first 6 months of the postoperative period. ${ }^{16}$ A stable IOL alignment was observed in both groups on PODs 1 and 30 in our cases. The postoperative IOL alignment was assessed with the toric enhancement software integrated in the iTrace aberrometer, which provides the actual and target axis of toric IOL as well as the amount of postoperative rotation required to achieve optimal results. ${ }^{17}$ Previous studies have employed retro-illumination photographs to evaluate toric IOL alignment. ${ }^{13,16}$ However, the procedure is time consuming, cumbersome, and may be subject to inter-observer variability. The iTrace aberrometer is an objective, simple, and comprehensive method to evaluate toric IOL alignment based on anatomical landmarks and the refractive state of the eye.

In our study, the mean deviation from the target axis was significantly less with Callisto eye and $\mathrm{Z}$ align than

Table 4 Correlation between postoperative visual quality and deviation from target axis of implantation of toric intraocular lens on postoperative days I and 30

\begin{tabular}{|c|c|c|c|c|}
\hline \multirow{2}{*}{$\begin{array}{l}\text { Correlation } \\
\text { parameters }\end{array}$} & \multicolumn{2}{|c|}{ Deviation from target axis, day I } & \multicolumn{2}{|c|}{ Deviation from target axis, day 30} \\
\hline & $\begin{array}{l}\text { Pearson's } \\
\text { correlation } \\
\text { coefficient }\end{array}$ & $p$-value & $\begin{array}{l}\text { Pearson's } \\
\text { correlation } \\
\text { coefficient }\end{array}$ & $p$-value \\
\hline Strehl ratio internal & -0.621 & $<0.001$ & -0.730 & $<0.001$ \\
\hline Strehl ratio total & -0.396 & $<0.001$ & -0.469 & $<0.001$ \\
\hline MTF internal 5 cpd & -0.788 & $<0.001$ & -0.795 & $<0.001$ \\
\hline MTF internal $10 \mathrm{cpd}$ & -0.710 & $<0.001$ & -0.794 & $<0.001$ \\
\hline MTF internal $15 \mathrm{cpd}$ & -0.671 & $<0.001$ & -0.772 & $<0.001$ \\
\hline MTF internal average & -0.771 & $<0.001$ & -0.828 & $<0.001$ \\
\hline MTF total $5 \mathrm{cpd}$ & -0.307 & 0.006 & -0.313 & 0.005 \\
\hline MTF total $10 \mathrm{cpd}$ & -0.322 & 0.004 & -0.206 & 0.067 \\
\hline MTF total $15 \mathrm{cpd}$ & -0.309 & 0.005 & -0.232 & 0.038 \\
\hline MTF total average & -0.320 & 0.004 & -0.239 & 0.033 \\
\hline
\end{tabular}

Abbreviations: MTF, modular transfer function; cpd, cycles per degree. 
with manual marking. The postoperative alignment of the toric IOL should be within $5^{\circ}$ of the intended axis to achieve optimal results. We observed mean deviation of less than $5^{\circ}$ with Callisto eye and $\mathrm{Z}$ align, whereas it was more than $5^{\circ}$ with manual marking. Only one previous study has compared toric IOL alignment with the Callisto eye and $\mathrm{Z}$ align and manual marking, and observed more precise IOL alignment with image-guided surgery with similar visual outcomes. ${ }^{7}$ The different image-guided systems may be equally efficacious in enhancing the accuracy of toric IOL alignment. Previous studies employing the Verion digital marker (Alcon Laboratories, Inc.) have reported it to result in more accurate toric IOL alignment than the manual marking technique. ${ }^{8,9}$ Callisto eye with $\mathrm{Z}$ align has the added advantage of being an integrated system, and it does not require an external attachment to the surgeon's eyepiece for visualization unlike other image-guided systems.

In our study, the visual outcomes between the two groups were comparable. In a 2014 ASCRS clinical survey, approximately one-third of the respondents indicated that a rotational error of $10^{\circ}$ or more may not significantly affect visual outcomes. ${ }^{18}$ We did not perform a vector analysis of the residual refractive cylinder, which is a limitation of our study.

We observed significantly better visual quality in the image-guided surgery group, as evidenced by higher internal Strehl ratio and MTF at all spatial frequencies. Moreover, there was a significant inverse correlation between parameters of visual quality and the deviation from target axis of implantation, with more precise toric IOL alignment associated with better MTF and Strehl ratio. Thus, even though the UDVA was comparable in both groups, a definite advantage in terms of visual quality was observed with image-guided surgery. This may be especially advantageous in implantation of premium IOLs such as multifocal toric IOLs, where even minor deviations from the target axis of implantation may lead to suboptimal visual acuity and quality.

Manual marking systems for toric IOL alignment give accurate results in experienced hands. ${ }^{13}$ The marking accuracy is subject to variability owing to the dependency on the marking skills of the surgeon and the associated learning curve. An element of torsion may persist while marking, resulting in rotational misalignment of the reference axis despite the best efforts of the surgeon. Manual marking systems tend to produce upward deviated reference marks, which may be attributed in part to the Bell's phenomenon. ${ }^{19}$ Moreover, the marks may fade if there is a gap between marking and the surgery or due to excessive tearing, necessitating repeat marking. Smearing of the ink is an additional source of error leading to imprecise alignment. All these limitations are effectively addressed by the image-guided systems. Image-guided systems are seamlessly integrated with the surgical microscope and the graphic overlay simplifies the process of toric IOL alignment and enhances its accuracy. The use of image-guided systems for toric IOL alignment eliminates the patient discomfort associated with manual marking and is convenient both for the patient as well as the surgeon. Preoperative and intraoperative image capture required in these systems may be time consuming; however, this disadvantage is outweighed by the ease of procedure and convenience. A more streamlined workflow and decreased surgical duration has been observed with the use of imageguided systems. ${ }^{7}$

In our study, a realignment of toric IOL was not needed in any case in both the groups. However, a larger series with a wide range of astigmatism as well as extremes of axial length is required to better assess the accuracy of toric IOL alignment by the two methods. Postoperative realignment of the toric IOL may be required in a few patients undergoing manual marking methods for toric IOL alignment in order to achieve optimal visual outcomes.

\section{Conclusion}

Image-guided systems are more accurate than conventional manual marking methods for the alignment of toric IOLs, and the enhanced precision may be especially advantageous in premium IOL implantation. Though the visual acuity is similar with both methods, image-guided surgery is associated with superior visual quality which correlates with the precision of IOL alignment. Ray tracing aberrometer is a precise and quick method to analyze postoperative toric IOL alignment and the refractive state of the eye.

\section{Author contributions}

All authors contributed toward data analysis, drafting and revising the paper and agree to be accountable for all aspects of the work.

\section{Disclosure}

The authors report no conflicts of interest in this work.

\section{References}

1. Hoffmann PC, Hütz WW. Analysis of biometry and prevalence data for corneal astigmatism in 23,239 eyes. $J$ Cataract Refract Surg. 2010;36: 1479-1485.

2. Ferrer-Blasco T, Montés-Micó R, Peixoto-de-Matos SC, GonzálezMéijome JM, Cerviño A. Prevalence of corneal astigmatism before cataract surgery. J Cataract Refract Surg. 2009;35:70-75. 
3. Collier Wakefield O, Annoh R, Nanavaty MA. Relationship between age, corneal astigmatism, and ocular dimensions with reference to astigmatism in eyes undergoing routine cataract surgery. Eye (Lond). 2016;30: $562-569$.

4. Shimizu K, Misawa A, Suzuki Y. Toric intraocular lenses: correcting astigmatism while controlling axis shift. J Cataract Refract Surg. 1994;20: 523-526.

5. Felipe A, Artigas JM, Díez-Ajenjo A, García-Domene C, Alcocer P. Residual astigmatism produced by toric intraocular lens rotation. J Cataract Refract Surg. 2011;37:1895-1901.

6. Visser N, Berendschot TT, Bauer NJ, Jurich J, Kersting O, Nuijts RM. Accuracy of toric intraocular lens implantation in cataract and refractive surgery. J Cataract Refract Surg. 2011;37:1394-1402.

7. Mayer WJ, Kreutzer T, Dirisamer M, et al. Comparison of visual outcomes, alignment accuracy, and surgical time between 2 methods of corneal marking for toric intraocular lens implantation. J Cataract Refract Surg. 2017;43:1281-1286.

8. Webers VSC, Bauer NJC, Visser N, et al. Image-guided system versus manual marking for toric intraocular lens alignment in cataract surgery. J Cataract Refract Surg. 2017;43:781-788.

9. Elhofi AH, Helaly HA. Comparison between digital and manual marking for toric intraocular lenses: a randomized trial. Medicine (Baltimore). 2015;94:e1618.

10. Osher RH. Iris fingerprinting: new method for improving accuracy in toric lens orientation. J Cataract Refract Surg. 2010;36:351-352.

11. Ligabue EA, Giordano C. Assessing visual quality with the point spread function using the NIDEK OPD-Scan II. J Refract Surg. 2009;25: S104-S109.
12. Woodcock MG, Lehmann R, Cionni RJ, Breen M, Scott MC. Intraoperative aberrometry versus standard preoperative biometry and a toric IOL calculator for bilateral toric IOL implantation with a femtosecond laser: one-month results. J Cataract Refract Surg. 2016;42:817-825.

13. Popp N, Hirnschall N, Maedel S, Findl O. Evaluation of 4 corneal astigmatic marking methods. J Cataract Refract Surg. 2012;38: 2094-2099.

14. Montes de Oca I, Kim EJ, Wang L, et al. Accuracy of toric intraocular lens axis alignment using a 3-dimensional computer-guided visualization system. J Cataract Refract Surg. 2016;42:550-555.

15. Onishi H, Torii H, Watanabe K, Tsubota K, Negishi K. Comparison of clinical outcomes among 3 marking methods for toric intraocular lens implantation. Jpn J Ophthalmol. 2016;60:142-149.

16. Koshy JJ, Nishi Y, Hirnschall N, et al. Rotational stability of a singlepiece toric acrylic intraocular lens. J Cataract Refract Surg. 2010;36: $1665-1670$.

17. Kaur M, Shaikh F, Falera R, Titiyal JS. Optimizing outcomes with toric intraocular lenses. Indian J Ophthalmol. 2017;65:1301-1313.

18. ASCRS Clinical Survey; 2014. Available from: http://eyeworld.org/ supplements/2014_ASCRS_clinical_survey.pdf. Accessed January 6, 2018.

19. Woo YJ, Lee H, Kim HS, Kim EK, Seo KY, Kim TI. Comparison of 3 marking techniques in preoperative assessment of toric intraocular lenses using a wavefront aberrometer. J Cataract Refract Surg. 2015;41:1232-1240.
Clinical Ophthalmology

\section{Publish your work in this journal}

Clinical Ophthalmology is an international, peer-reviewed journal covering all subspecialties within ophthalmology. Key topics include: Optometry; Visual science; Pharmacology and drug therapy in eye diseases; Basic Sciences; Primary and Secondary eye care; Patient Safety and Quality of Care Improvements. This journal is indexed on

Submit your manuscript here: http://www.dovepress.com/clinical-ophthalmology-journal

\section{Dovepress}

PubMed Central and CAS, and is the official journal of The Society of Clinical Ophthalmology (SCO). The manuscript management system is completely online and includes a very quick and fair peer-review system, which is all easy to use. Visit http://www.dovepress.com/ testimonials.php to read real quotes from published authors. 